
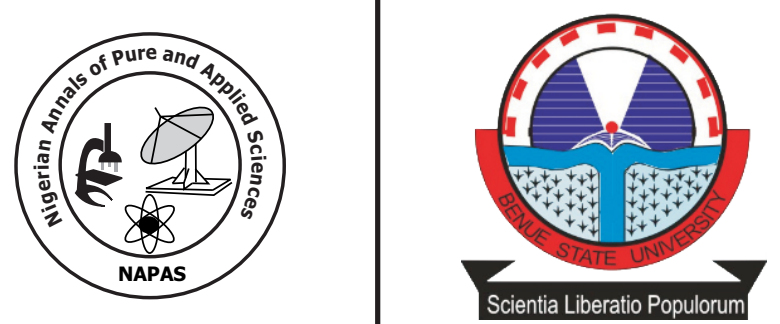

\title{
Mathematical Evaluation of the Effect of Agrochemicals on Human Health
}

\author{
Agaba, G.0. \\ Department of Mathematics and Computer Science, \\ Benue State University, Makurdi, Nigeria \\ *Corresponding author: omecheagaba@gmail.com
}

\section{Abstract}

The fast growing economical and political needs and demands for increase agricultural activities and produces in most parts of the world necessitate the need for a careful evaluation of the general crop cultivation processes and the increasing usage of diverse toxic chemicals in the form of pesticides, fertilizers, etc. in the agricultural sector to control pests and weeds to improve soil nutrients during cultivation and in some cases, for preservation of cultivated crops. Agrochemicals are used in a manner that suggests farmers do not take into cognisance the fact that residue of the chemicals which is harmful to man is always left on such foods. This paper applies a mathematical model to examine the impact of agrochemicals on human health by using the conventional principle of an SEIRS epidemic model. From the overall outcome, it was observed that the consumption of these hazardous plants before the elapse of the incubation (waiting) period affect the human health negatively. Consequently, the need for a wake up call to all farmers, agricultural workers, Government and Non-Governmental monitoring agencies to arise to the task of saving human lives from these toxic chemical residues in agricultural produces.

Key word: Plants, Toxic chemicals, Incubating period, Human health 


\section{Introduction}

Agriculture is gradually taking the lead focus in the world's economy. Consequently, the greatest challenge of our present day agriculture is centred on how it can feed the fast growing population of the world and to restore the natural resources (Kumari et al., 2014). According to Kumari et al. (2014), the expected goal is to double the required quantity of global food production required to handle the needs of the general masses by 2020 and to maintain the current consumption rate of the entire population. In Mexico, it is on record that one of the most important lifestyles of the citizens is hinged on agricultural activities, noting that as $20 \%$ of the economically active population are agricultural driven (Salas et al., 2000). In light of the aforementioned, the use of farm chemicals such as fertilizers and pesticides among other chemicals have been on the increase (Berg and Tam, 2012; Bernhardt et al., 2017; Kumari et al., 2014; Moser et al., 2017; Roslund, 2015; Schwarzenbach et al., 2006; Tompkins and Bird, 2004) even though their application had yielded much increase in crop productivity and its production, the adverse toxic effects on the cultivated land, the produces and human health as an end user/receiver have also increased proportionately (Blair et al., 2014; Kumari et al., 2014; Salas et al., 2000; WHO, 2008).

The excessive usage of these chemicals are by no means of positive impact instead they have caused great havoc to the immediate environment as well as human health indirectly through air, water, drinking water and food (most especially the consumption of recently fumigated food products like vegetables) (Kumari et al., 2014; Roslund, 2015; Salas et al., 2000; Talukdar et al., 2003; WHO, 2008). The World Health Organization warms that families who consume food supplied or harvested directly from the farm/fields are likely to be more exposed to pesticides due to the poor monitoring or control of residues of pesticides in our locally produced foods (WHO, 2008).

Agrochemicals, which include herbicides, insecticides fungicides, fumigants, among other chemicals (Blair et al., 2014; Roslund, 2015; WHO, 2008) are mostly used on farms as means of controlling, eliminating or eradicating diseases, vectors and pests, weeds etc. from destroying the crops and also in some cases to add nutrients to the soil (Alavanja et al., 2004; Blair et al., 2014; Moser et al., 2017; Roslund, 2015; Salas et al., 2000; UNEP, 2014; WHO, 2008). According to Roslund (2015), "what makes pesticides effective against disease and pest makes them hazardous to humans and to the environment and therefore causes harm to non-target species". The World Health Organization (WHO) has on record that about 500,000 to 1 million persons are poisoned with pesticides yearly out of which about 5,000 to 20,000 loss their lives. This they tied to the low educational level of the agricultural workers has they have no or less knowledge of the chemicals and their applications (Salas et al., 2000).

Aside air pollution which have been considered to constitute serious hazard to human health and have been classified globally to be among the 10 most significant risk factors for public health, land and food pollution have also in the past years threaten the well being of humans (Agaba, 2013; Agaba, 2014; Blair et al., 2014; Kumari et al., 2014; Lim et al., 2012; Prank et al., 2016; Webster et al., 2005). In as much as it is expected that during pesticide production and its application on farms (crops) humans are exposed occupationally, "the general human population can also be exposed through drift, contamination of water and food supplies, and biological concentration through the food chain" (Moser et al., 2017). According to Rodin and Egan (1989), the concentration of the chemical substances determines the chemical reaction rate, since the rate of chemical reaction varies in proportion to the concentration of the substances.

An evaluation of the amount of daily intake of toxic residues of agricultural chemicals in human diet was carried out for some selected countries, the result shows that India had the highest average dietary intake of pesticides residues in $\mathrm{mg} /$ day/person, Europe was the next in line while USA had the least value of pesticides residue consumption (Kumari et al., 2014). Consequently, it was noted that India had the highest likelihood of human ill-health resulting from the intake of these chemical residues.

Record has it that the consumption of food grown with chemical, either meant for pest or weeds control or to improve agricultural yields, are responsible for various deteriorating health hazards both in animals and human beings. Some of the effects resulting from such act are: lungs irritation, central nervous system disorder, depression, oral acetomatism, cancer, birth defeats, growth retardation, kidney and immune system damage etc. (Blair et al., 2014; Kumari et al., 2014). According to Agaba (2014), food containing hazardous substances are term unsafe for human consumption since they can cause illness instantaneously or increases the risk of chronic diseases. Udeze in 2011 identify wrong use of agrochemicals as one of the causes of food poisoning (Udeze, 2011). In support of the above 
statement, chemical residues in grains were discovered, within the same period of time, as source of food poisoning during a training workshop held in Abuja, Nigeria (Agaba, 2014).

In order to reduce the adverse effect of these chemicals on the cultivated crops, the environments and the consumers, Kumari et al. (2014) opined that the dependence on chemical inputs on agricultural activities should be reduced and this they believed is possible only through the ecofriendly approaches of farming system. While Webster et al. (2005) suggested the idea of modelling and monitoring as they uphold the concept that monitoring programs contribute to the scientific understanding of environmental process and through such processes, better models can be developed with the desire to achieve the highest benefit of improved chemical management. In 2005, Huan et al. (see details in Roslund, 2015) carried out a study and actually discovered that the reduction in the quantity of pesticides used resulted in a corresponding reduction in the labour and exposure to pesticide poisoning.

This paper studies the implications of consuming unhealthy plants on human health by deriving a mathematical model that examine the impact of hazardous chemicals indirectly on human health, that is, through the consumption of plants sprayed with toxic chemical before the expected due time required for the plant to be declare fit for consumption. This was done using the principle of the conventional SEIRS epidemic model to represent the plant population dynamics with an additional class representing the human population and how they relate with the plant population. The outline of the paper consists of five Sections with the next Section centred on the model derivation while the analyses of the various steady states and their stability were carried out in Section 3. In Section 4, the paper considered the numerical evaluation of the model equation and finally the fifth Section covered the discussion of the study.

\section{Model derivation}

Considering a cultivated piece of land having a total number of crops or plants denoted by $x$ which is assumed to grow logistically with $r$ representing the growth rate of the plant and $k x$ the carrying capacity of the cultivated land. It is also assumed that the plants were sprayed with a chemical either for pest or weed control, or to improve soil nutrients and/or for preservation. The sprayed plants are considered to be harmful to human health as a result of the chemical composition until an elapse time interval known as the incubation period or waiting period.
Consequently, the plants are fit for consumption only after the waiting time has elapsed.

Therefore, let $z$ denote the level of chemical application or composition sprayed on the farmland (plants) and the population $x$ represents the healthy plants while $u$ denotes the incubated plant population and $y$ represents the harmful plant populations removed from the total number of incubated plants before the elapsed of the waiting period. Assuming that the human population denoted by $s$ grows logistically with a carrying capacity, $k s$ and the birth and death rates are represented by $b$ and $\phi$ respectively, the model equation is therefore obtain as

$$
\begin{aligned}
& \frac{d x}{d t}=r\left(1-\frac{x}{k_{x}}\right) x-\beta x z+\eta q u \\
& \frac{d u}{d t}=\beta x z-\eta u \\
& \frac{d y}{d t}=\eta p u-(\alpha+\delta) y \\
& \frac{d z}{d t}=\omega-\lambda z \\
& \frac{d s}{d t}=b\left(1-\frac{s}{k_{s}}\right) s-\phi s
\end{aligned}
$$

where $\beta$ represents the rate at which the chemical is applied on the plant, indicating the contact rate between the healthy plants and the chemical sprayed $\eta$ denotes the removal rate of plants from the incubated population with certain proportion of these plants becoming healthy and others unhealthy or harmful. The paramete $q$ denotes the proportion of the incubated plants that recovered from the effect of the applied chemical after the elapsed of the waiting or incubated period, that is, after the time taken for the chemicals to wane off from the plant without leaving any residue and thus the plants become consumable or healthy. The incubating period of the chemical effect on the plant is considered to be the time from the application of the chemical to the time it fades off leaving the plants fit/healthy for consumption. Consequently, $p(p+q=$ 1 ) represents the proportion of the plants removed from the incubated plants before the complete duration of time required for the plants to be incubated. Therefore, any plant consumed from the proportion of unhealthy or harmful plants is considered harmful and deadly to the consumers.

The parameter $\lambda$ represents the rate at which the chemical applied losses it strength/toxicity or in some rare cases when it is washed off as a result of rainfall and other human factors respectively, it implies that $1 / \lambda$ is the average duration of the chemical strength. While $\omega$ is the amount of the chemical concentrate used in the process of pest/weed control or nutrient enhancement. The rate 
at which humans consume the unhealthy plants is denoted by the parameter $\alpha$ and the death rate of the plants which in most cases could be as a result of the very high chemical concentration or composition is represented by $\delta$ respectively.

The amount of healthy plant consumed can be taken to increase the well being of the human population thereby affecting the population size positively. Thus, the death rate of human population reduces with the increase consumption of healthy plants while the reverse is the case when unhealthy (harmful) plants are consumed. As more harmful plants are consumed by the human population, the human death rate is therefore enhanced as a result of the effect and reactions of the toxic chemicals being consumed. Consequently, we obtain the following equation accounting for the corresponding effect on the human populations.

$\phi_{y}(\alpha)=\phi+\frac{\varepsilon \alpha}{1+\alpha}$

Equation 2 indicates the corresponding increment in human death rate as a result of the increase in the consumption of harmful plants. The parameter $\phi$ represents the natural death rate of humans in the absence of the effect of farm chemicals while for any small consumption of harmful plants, the death rate grows linearly with $\alpha$ and saturates at some steady level when the consumption gets high, this is because it is considered to generate some repulse effect when its impact become too much or when the entire affected plants are consumed.

Consequently, the above modification gives the required model as

$$
\begin{aligned}
& \frac{d x}{d t}=r\left(1-\frac{x}{k_{x}}\right) x-\beta x z+\eta q u \\
& \frac{d u}{d t}=\beta x z-\eta u \\
& \frac{d y}{d t}=\eta p u-(\alpha+\delta) y \\
& \frac{d z}{d t}=\omega-\lambda z \\
& \frac{d s}{d t}=b\left(1-\frac{s}{k_{s}}\right) s-\phi_{y}(\alpha) s
\end{aligned}
$$

with $p+q=1 \quad \phi_{y}(\alpha)=\phi+\frac{\varepsilon \alpha}{1+\alpha}$ and the initial conditions: $x(0)>0 u(0) \geq y(0)=0 z(0) \geq 0 s(0)>0$ defined within the region

$D=\left\{(x, u, y, z, s) \in R_{+}^{s}: 0 \leq x, u, y \leq k_{z} 0 \leq z \leq \frac{\omega}{\lambda}, 0 \leq s \leq k_{z}\right\}$.

The model flow diagram is captured in Figure 1.

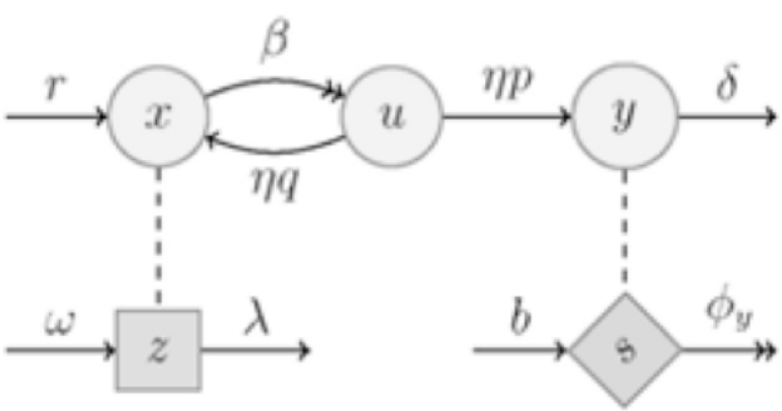

Figure 1: Model diagram: dynamics of transitions associated with the various groups in equation 3 . Solid line with double-head arrows indicate transition processes influenced by contacts associated with the chemical, those with single-head arrows indicate processes that are independent of contact, while dash lines indicate processes leading to contact transitions. Healthy, incubated and harmful plant population are denoted by $x, u, y$, while $s$ and $z$ denote the amount of chemical application and human population respectively.

\section{The steady states and their stability}

The model equation has three steady states: the first is the plant-free steady state, $E_{0}$, which represents the state before the planting of crops wherein only human population exists having the chemical product on standby; the second is the hazard-free steady state, $E_{1}$ that is, the steady state free of chemical application on the plants denoting a scenario where farmers are yet to apply any pest or weed control chemical, and/or fertilizer etc. on the planted crops; and lastly the endemic steady state, $E^{*}$ representing the stage with the hazardous efffect of chemical application on both the plants and humans.

The plant-free steady state is obtain as

$E_{0}=[\hat{x}, \hat{u}, \hat{y}, \hat{z}, \hat{s}]=\left[0,0,0, \frac{\omega}{\lambda}, k_{s}\left(1-\frac{\phi}{b}\right)\right]$

and has the characteristics equation

$(\alpha+\delta+\mu)(\lambda+\mu)\left(\frac{b \hat{s}}{k_{s}}+\mu\right)\left[\left(r-\frac{\beta \omega}{\lambda}-\mu\right)(\eta+\mu)+\frac{\beta \omega \eta q}{\lambda}\right]=0$,

which implies

$(\alpha+\delta+\mu)(\lambda+\mu)\left(\frac{b \hat{s}}{k_{s}}+\mu\right)\left[\mu^{2}+\mu\left(\frac{\beta \omega}{\lambda}+\eta-r\right)+\frac{\beta \omega \eta p}{\lambda}-\eta r\right]=0$

with $\hat{s}=k_{s}\left(1-\frac{\phi}{b}\right)>0$ and $p=1-q$

Consequently, this conote that the steady state is stable provided

$\frac{\beta \omega}{\lambda}+\eta-r>0$ and $\frac{\beta \omega \eta p}{\lambda}-\eta r>0$.

But $\beta \omega+\lambda \eta>\lambda r$ is always satisfied if $\beta \omega p>\lambda r$ Hence, if the threshold value is defined as

$R_{0}=\frac{\lambda r}{\beta \omega p}$,

then it gives the following result. 
Theorem 1. The plant-free steady state $E_{0}$ is stable provided $R_{0}<1$ unstable if $R_{0}>1$ and undergoes steady state bifurcation when $R_{0}=1$

The hazard-free equilibrium state $E_{1}$ is obtained as

$E_{1}=[\bar{x}, \bar{u}, \bar{y}, \bar{z}, \bar{s}]=\left[k_{x}, 0,0,0, k_{s}\left(1-\frac{\phi}{b}\right)\right]$

and from the Jacobian matrix the characteristics equation is generated as

$(r+\mu)(\eta+\mu)(\alpha+\delta+\mu)(\lambda+\mu)\left(\frac{b \bar{s}}{k_{s}}+\mu\right)=0$,

which implies that it is always stable whenever it exists, thus the following result.

Theorem 2. The hazard-free equilibrium state $E_{1}$ of the model equation 3 is always stable whenever it exists.

The endemic steady state $E^{*}$ of the model equation is defined as

$E^{*}=\left[x^{*}, u^{*}, y^{*}, z^{*}, s^{*}\right]$,

where

$$
\begin{aligned}
x^{*}=k_{x}\left(1-\frac{\beta \omega p}{\lambda r}\right), \quad u^{*}=\frac{\beta \omega x^{*}}{\lambda \eta}, \quad y^{*}=\frac{\beta \omega p x^{*}}{\lambda(\alpha+\delta)}, \quad z^{*}=\frac{\omega}{\lambda}, \\
s^{*}=k_{s}\left(1-\frac{\phi_{y}(\alpha)}{b}\right) \quad \text { with } \phi_{y}(\alpha)=\phi+\frac{\varepsilon \alpha}{1+\alpha}
\end{aligned}
$$

and the endemic state is feasible only when $R_{0}>1$ While its stability is obtain using the Jacobian matrix

$I^{*}=\left(\begin{array}{ccccc}r\left(1-\frac{2 x^{*}}{k_{x}}\right)-\beta z^{*} & \eta q & 0 & -\beta x^{*} & 0 \\ \beta z^{*} & -\eta & 0 & \beta x^{*} & 0 \\ 0 & \eta p & -(\alpha+\delta) & 0 & 0 \\ 0 & 0 & 0 & -\lambda & 0 \\ 0 & 0 & 0 & 0 & -\frac{b s^{*}}{k_{s}}\end{array}\right)$

The characteristics equation of

$(\alpha+\delta+\mu)(\lambda+\mu)\left(\frac{b s^{*}}{k_{s}}+\mu\right)\left[\left(r-\frac{2 r x^{*}}{k_{x}}-\beta z^{*}-\mu\right)(\eta+\mu)+\eta q \beta z^{*}\right]=0$

$\Rightarrow(\alpha+\delta+\mu)(\lambda+\mu)\left(\frac{b s^{*}}{k_{s}}+\mu\right)\left[\mu^{2}+\mu\left(\eta+\frac{r x^{*}}{k_{x}}+\frac{\beta \omega q}{\lambda}\right)+\frac{r \eta x^{*}}{k_{x}}\right]=0$,

with $x^{*}, z^{*}$, and $s^{*}$ as defined in equation 5 . Hence, it generates the following result.

Theorem 3. The endemic steady state $E^{*}$ is only feasible when $R 0>1$ and is always stable whenever it exists.

Remarks. Note that if the threshold value $R 0<1$ the system of equations 3 has a plant-free steady state $E_{0}$ which is stable but become unstable when $R 0>1$ and therefore transit to an endemic steady state that is also stable whenever it exists. Furthermore, as Ro $\rightarrow \infty$ the endemic steady state transit to the hazardfree steady state $E_{1}$ this is because the chemical losses its strength over time. This signifies that from equation 4 we have the following

$\lim _{\omega \rightarrow 0} R_{0}=\lim _{\omega \rightarrow 0} \frac{\lambda r}{\beta \omega p}=\infty \Rightarrow R_{0} \rightarrow \infty$ as $\omega \rightarrow 0$

and

$\lim _{\omega \rightarrow \infty} R_{0}=\lim _{\omega \rightarrow \infty} \frac{\lambda r}{\beta \omega p}=0 \Rightarrow R_{0} \rightarrow 0$ as $\omega \rightarrow \infty$.

Similarly,

$\lim _{\beta \rightarrow 0} R_{0}=\infty \Rightarrow \beta \rightarrow 0, R_{0} \rightarrow \infty$ and $\lim _{\beta \rightarrow \infty} R_{0}=0 \Rightarrow \beta \rightarrow \infty, R_{0} \rightarrow 0$,

and in the same light, the limit give

$R_{0} \rightarrow 0$ as $\lambda \rightarrow 0$ and $R_{0} \rightarrow \infty$ as $\lambda \rightarrow \infty$.

By implication, as $R_{0} \rightarrow \infty$ either $\beta \rightarrow 0$ and/or $\lambda$ $\rightarrow \infty$ indicating that as the applied chemical losses its toxicity with time; with no further application of the chemical on the plants, the entire crops will eventually become healthy plants again after an elapsed time interval resulting to the hazard-free steady state E1. Consequent upon the above results, it is observed that for $R 0<1$ the system exhibits a stable plant-free steady state EO while for $R 0>1$ it has an endemic steady state $E^{*}$ that is stable and as $R_{0} \rightarrow \infty$ it become an hazardfree steady state $E 1$ that is also stable.

\section{Numerical study of the model}

A numerical simulation with varied parameter values were carried out on the model equation in order to ascertain the level of the parameter impact on the dynamics. Figure 2 shows the stability region of the various steady states of the model with respect to some varied parameters. From the result captured in Figure 2, it is noted that the region below the curve line gives the region where $R_{0}>1$, that is, the stable endemic steady state and also in this region, the plant-free steady state is unstable. While the region above the curve line indicates the region where $R_{0}<1$, which implies that in this region the endemic steady state is not feasible (do not exists) but the plant-free steady state is stable. 
(a)

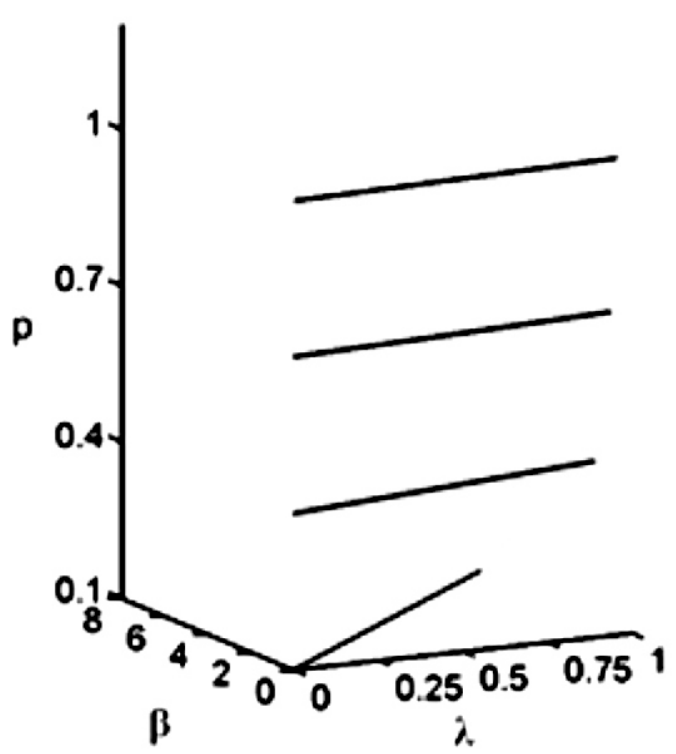

(b)

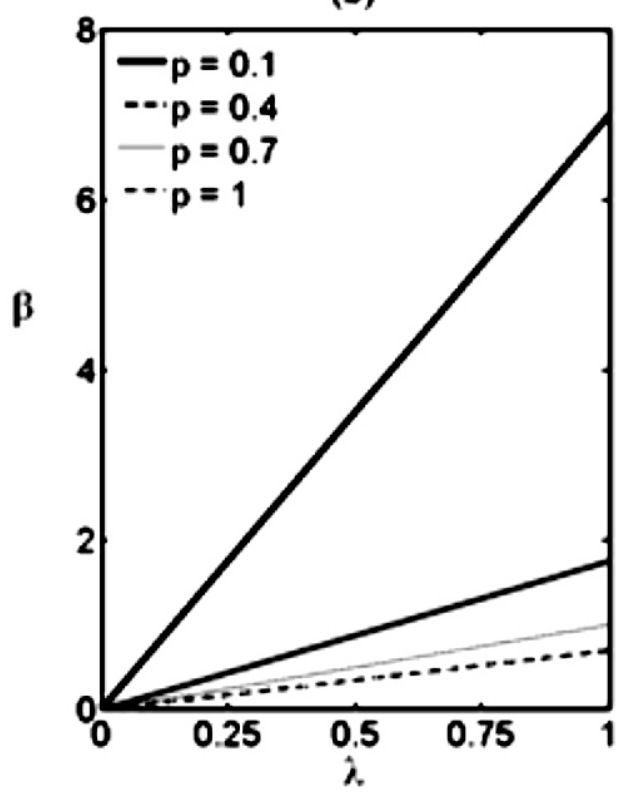

Figure 2: Stability region of the model in (a) three dimensional plot (b) two dimensional plot. Parameter values are $r=0.14$ and $\omega=0.2$.

Evaluating the system of equations 3 numerically generated the dynamics for the various population classes as represented in Figure 3 to 5 . Figure 3 represents the dynamic of the model equations when $\mathrm{R}_{0}<1$, that is, it shows the dynamic of the plant - free steady state of the model, while for the endemic steady state where $\mathrm{R}_{0}>1$ the result is captured in Figure 4 and as $\mathrm{R}_{0} \rightarrow \infty$, , the result in Figure 5 represents the dynamics of the hazard free steady state showing the various population. (a)

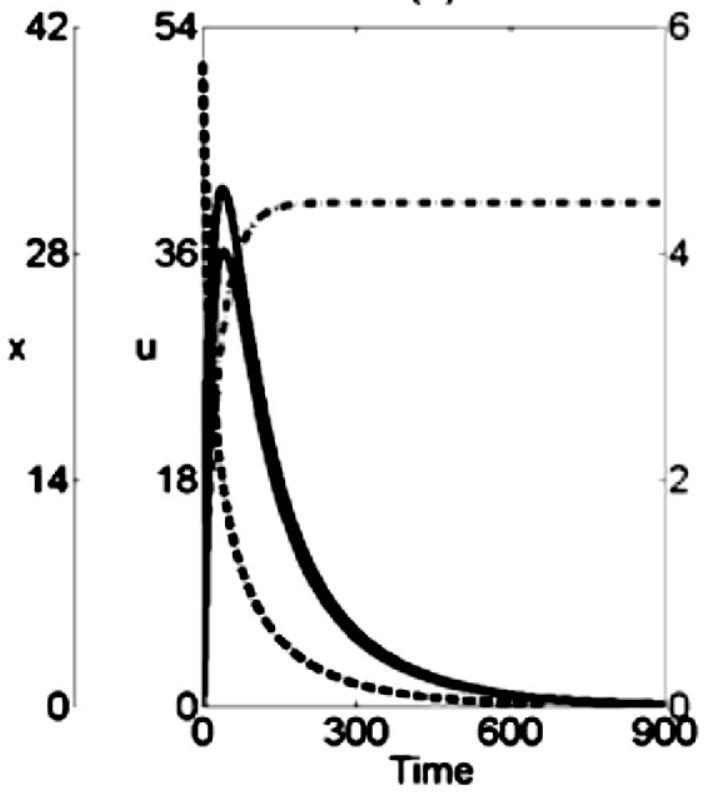

(b)

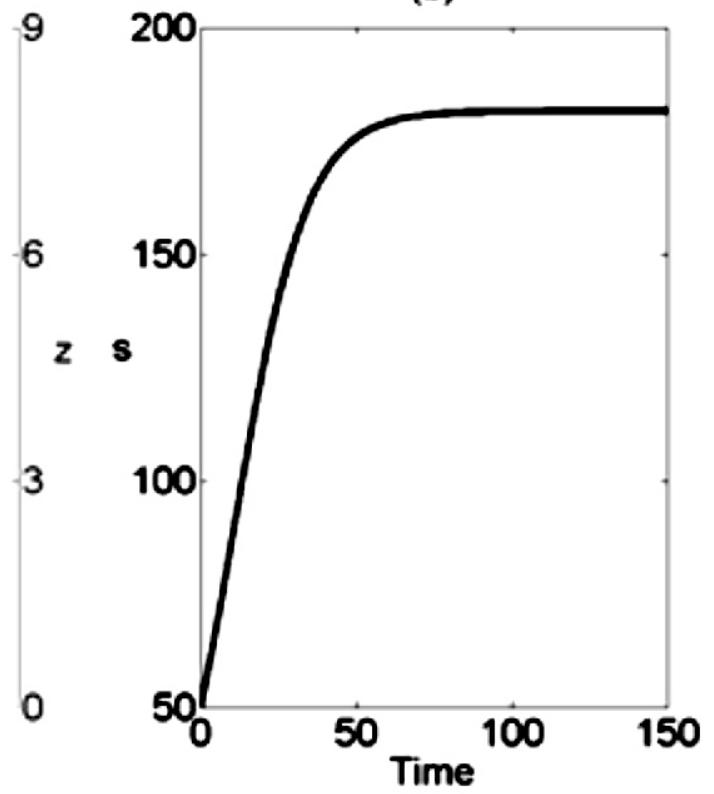

Figure 3: Dynamics of the model with $\mathrm{R}_{0}<1 \mathrm{R}_{0}=0.8750$ ) (a) plant population and quantity of chemical concentration (b) human population. The parameter values are $r-0.14, b=0.24, \eta=0.1, \delta=0.008, p=$ $0.4, q=0.6, \lambda=0.03, \alpha=0.4, \beta=0.06 . \phi=0.15, \varepsilon=0.01, \omega=0.02, k_{x}=50, k_{s}=500$. 
(a)

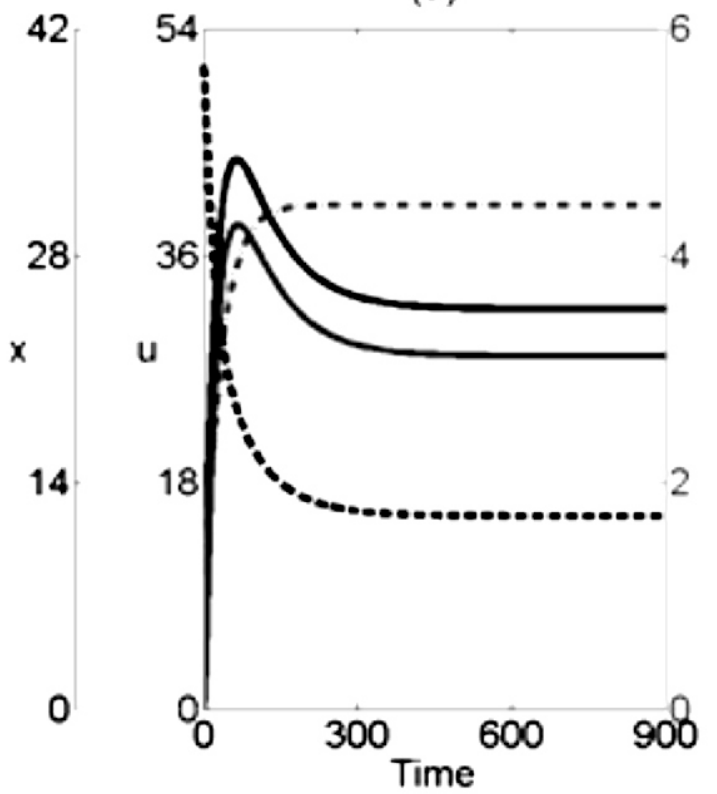

(b)

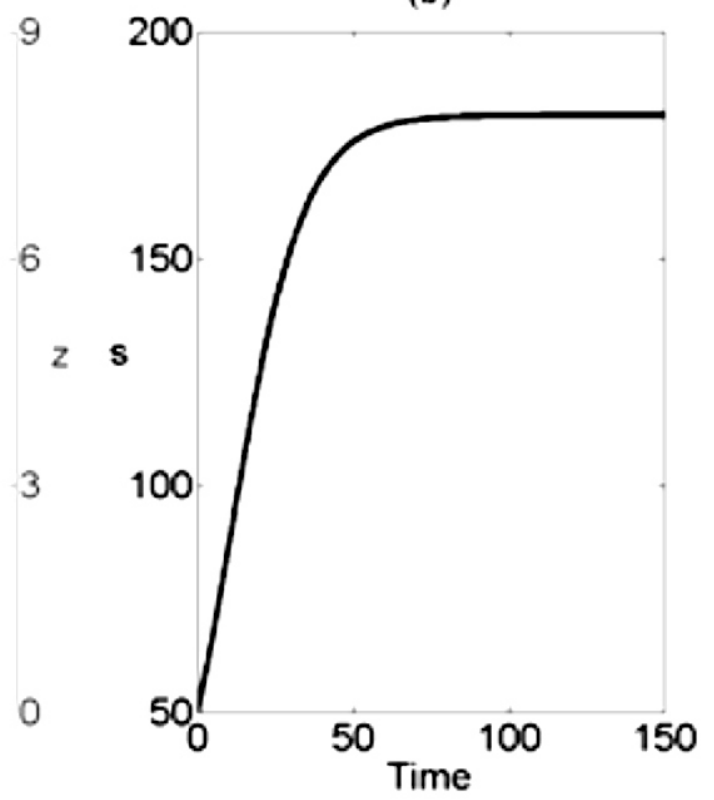

Figure 4: Dynamics of the model with $\mathrm{R}_{0}>1\left(\mathrm{R}_{0}=1.3125\right)$ (a) plant population and quantity of chemical concentration (b) human population. The parameter values are the same with those in Figure 3 except that $\beta=$ 0.04 .

(a)

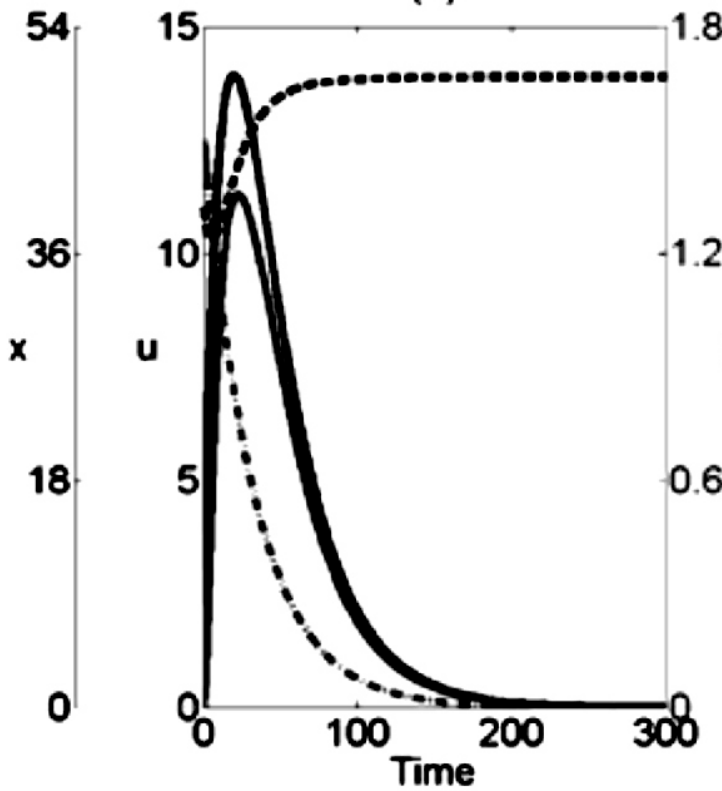

(b)

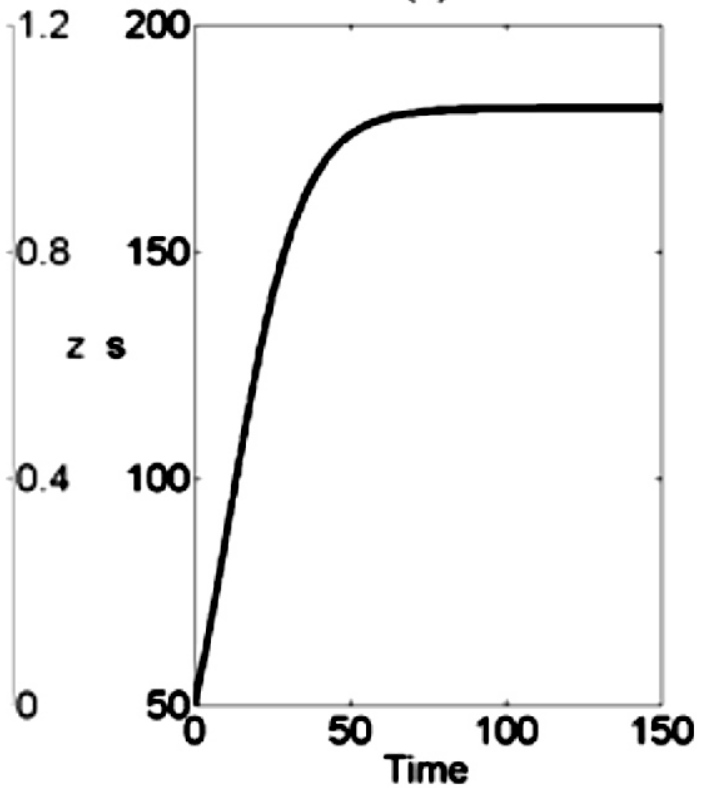

Figure 5: Dynamics of the model as $\mathrm{R}_{0} \rightarrow \infty$ (a) plant population and quantity of chemical concentration (b) human population. Aside $\omega=0$, all other parameter values are as stated in Figure 3.

A general representation of the steady states is given in Figure 6 wherein we have the various dynamics of all the respective populations plotted against the threshold value, $R_{0}$. The result shows the transitions from one steady state to the other, that is, from $E_{0}$ to $E^{*}$ and then finally to $E_{1}$ confirming the statement under Remarks. Figure $6 \mathrm{~b}$ gives a zoom position of the result to clearly show the behaviour of the dynamics as regard the various classes of population within the interval $[0,6]$ (i.e $R_{0} \in 0,6$ 
(a)

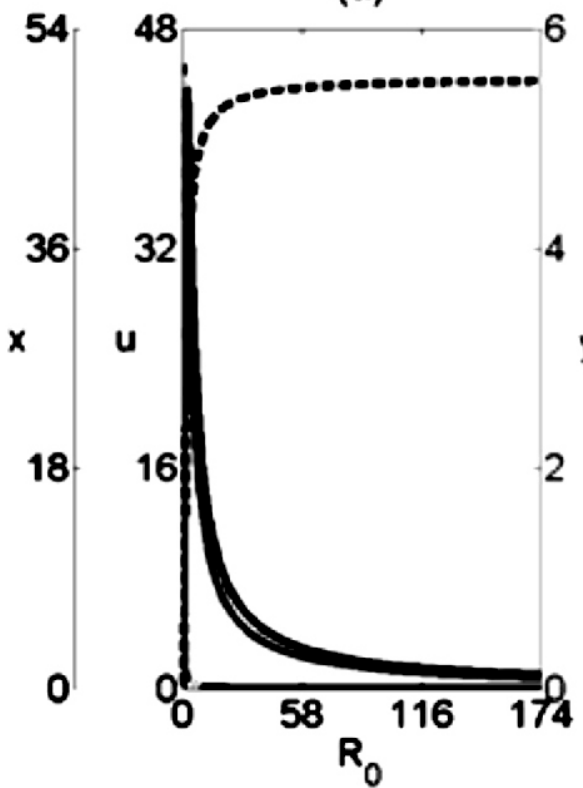

(b)

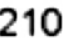

210

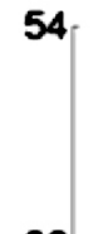

Figure 6: Graph of the population dynamics against $R_{0}$ (a) plant population and quantity of chemical concentration (b) a zoom position of (a) showing the dynamics within the interval $[0,6]$. Parameter values are the same with those in Figure 4 but the value of $\lambda$ was varied.

Further evaluation of the model equation with varied values of the parameter $\alpha$ was carrried out in order to determine the impact of the

(a)

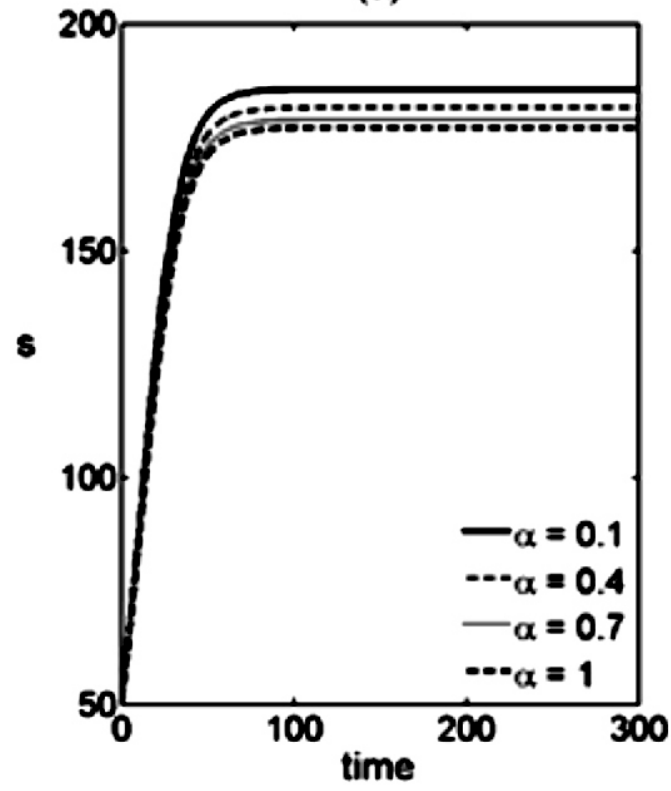

consumption rate of harmful or hazardous plants on human health (population). The extracted result is shown in Figure 7.

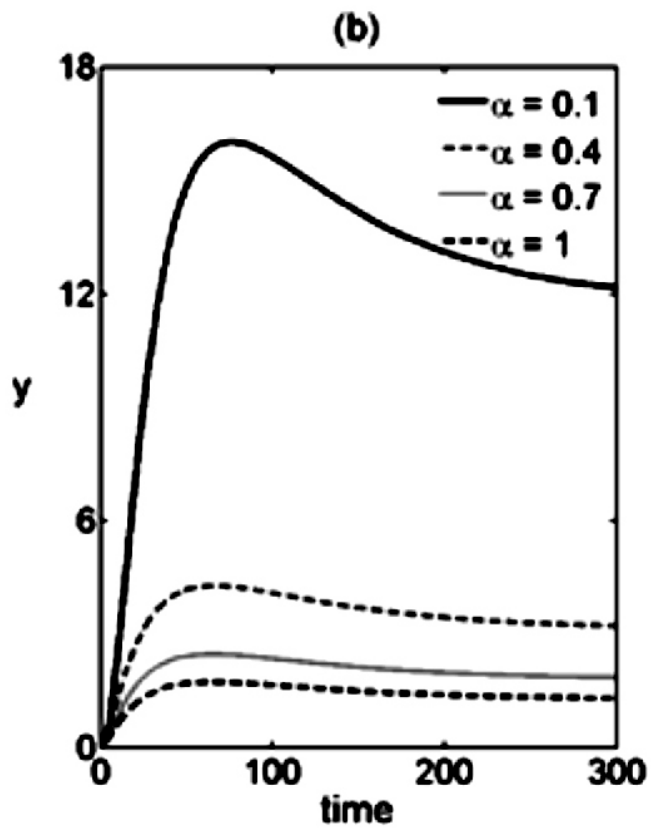

Figure 7: Graph showing the impact of the parameter, $\alpha$ on (a) the human population and (b) the corresponding effect on hazardous plant population. The parameter values are the same with those used to obtain Figure 4. But in this case, multiple values of $\alpha$ was used.

\section{Discussion}

Consequent upon the over all outcome of the analytical and numerical analyses of the model, it suffice to say that the threshold value $R_{0}$ plays significant role in the stability analysis of the model. It was clearly shown that the system has three steady states: the plant-free steady state $E_{0}$, endemic steady state $E^{*}$ and the hazard-free steady state $E_{1}$. The results of the numerical simulations confirmed the analytical analyses of the model equation 3 which indicate that the stability of the various steady states depend on the critical value of the threshold $R_{0}$ Details of which were given under Remarks. The numerical results captured by Figures 3 to 5 complement Theorems 1, 2 and 3 stated earlier. 
From Figure 7, one can clearly see the impact of consuming unhealthy plant, that is, the result shows that the consumption of hazardous plant has general negative health implications on human population. This implies that the general consumption of sprayed plants or crops with toxic chemical residues without waiting for the stipulated time recommended that the plant will be fit for consumption (i.e before the incubation period elapsed) affect the human health which in most cases leads to ill-health and eventually death. The outcome of this study shows that as the consumption rate of hazardous plants increases, it causes a corresponding increase in the death rate and consequently decreases the total number of the human population.

In essence, the overall outcome show the importance of the waiting or incubation period as recommended for each chemical and the need for them to be duly observe or comply with so as to avert any havoc that might emanate from such negligence. Therefore, it is of paramount importance for farmers, all agricultural workers and Government monitoring teams/agents or agencies to be educated on the need of ensuring that plants are not harvested and shipped for consumption before the elapsed waiting period of any toxic chemical used during cultivation processes.

\section{References}

Agaba, G.O. (2013). Mathematical modeling for the control of water pollution in Nigeria. In Fwatshak, S., Brown, G.N.M, and Agwuele, A.O., editors, The Challenge of Globalization, Environmental Degradation and Terrorism in Africa. Mediateam IT Educational Publishers, Berlin-Germany.

Agaba, G.O. (2014). Food adulteration: A threat to food security. In Owete, K.I., Emmanuel, M., Danfulani, U.H.D., Fwatshak, S., and Agwuele, A.O., editors, Freedom, Selfdetermination and Growth in Africa. Mediateam IT Educational Publishers, Berlin-Germany.

Alavanja, M.C., Hoppin, J.A., and Kamel, F. (2004). Health effects of chronic pesticide exposure: Cancer and neurotoxicity. Public Health, 25:155-197.

Berg, H., and Tam, N.T. (2012). Use of pesticides and attitude to pest management strategies among rice and rice-fish farmers in the Mekong Delta, Vietnam. International Journal of Pest Management, 58:153-164.

Bernhardt, E.S., Rosi, E.J., and Gessner, M.O. (2017). Synthetic chemicals as agents of global change. Frontiers in Ecology and the
Environment, 10.1002/fee.1450, 15:84-90.

Blair, A., Ritz, B., Wesseling, C., and Freeman, L.B. (2014). Pesticides and human health. Occupational and Environmental medicine, http://doi:10.1136/oemed-2014-102454.

Kumari, K.A., Kumar, K.N.R., and Rao, C.H.N. (2014). Adverse effects of chemical fertilizers and pesticides on human health and environment. Journal of Chemical and Pharmaceutical Sciences, 3:150-151.

Lim, S.S. et al. (2010). A comparative risk assessment of burben of disease and injury attributable to 67 risk factors and risk factor clusters in 21 regions, 1990 - 2010: A systematic analysis for the Global Burden of Disease Study 2010. doi:10.1016/S01406736(12)61766-8, 380:2224-2260.

Moser, A., Wemyss, D., Scheidegger, R., Fenicia, F., Honti, M., and Stamm, C. (2017). Modelling biocide and herbicide concentrations in catchments of the rhine basin. Hydro. Earth Syst. Sci. Discuss., https://doi.org/10.5194/hess-2017-628, pages 1-36.

Prank, M., et al. (2016). Evaluation of the performance of four chemical transport models in predicting the aerosol chemical composition in europe in 2005. Atmos. Chem. Phys., 16:6041-6070.

Rodin, E.Y., and Egan, K. (1989). Mathematical modelling of the rate of chemical reactions. Mathl. Comput. Modelling, 12:1707-1713.

Roslund, G. (2015). Rice farming models: A comparison between conventional and large-scale rice farmer's agricultural work practice in the Mekong Delta, Vietnam. B.Sc. Thesis of Science in Environmental Health, Umea University.

Salas, B.V., Duran, E.I.G., and Wiener, M.S. (2000). Impact of pesticides used on human health in Mexico: A review. Reviews on Environmental Health, 15:399-412.

Schwarzenbach, R.P., Escher, B.I., Fenner, K., Hofstetter, T.B., Johnson, C.A., Von Gunten, U., and Wehrli, B. (2006). The challenge of micropollutants in aquatic systems. Science, 10.1126/science. 1127291,313:1072-1077.

Talukdar, N., Thakuria, D., and Goswani, C. (2003). Organic farming and quality of organic food. In Borah, R.C., Talukdar, A., Kataky, J.C.S., Unni, B.G., Modi, M.K., and Deka, P.C., editors, Bioprospecting of commercially important plants. Indian Society of Agricultural Biochemists, Jorhat Chapter, Assam, India.

Tompkins, P., and Bird, C. (2004). Chemicals, 
plants and man: The organic farming residue. In Secret life of plants: A fascinating account of the physical, emotional and relations between plants and man. Rupa and Company.

Udeze, A. (2011). NAFDAC sensitises farmers on food poisoning. The Nation, www.thenationlineng.net/2011/index.php/ne wsextra/20245-nafdac-sensitises-farmers-onfood-poisoning-html.

UNEP (2014). Pesticides. http://www.unep.org/ chemicalsandwaste/UNEPsWork/Pesticide s/ tabid/298/Default.aspx.
Webster, E., Mackay, W.F., Arnot, D. J., Gobas, F., Gouin, T., Hubbarde, J., and Bonnell, M. (2005). Development and application of models of chemical fate in Canada: Modelling guidance document. Report to Environment Canada, Canadian Environmental Modelling Network, Trend University, Canada.

World Health Organization (WHO) (2008). Pesticides: Children's health and the environment. WHO Training Package for the Health Sector, www.who.int/ceh. 